\title{
Du NÉOlithiQue FINAL AU BRONZE ANCIEN : LES SÉPULTURES INDIVIDUELLES CAMPANIFORMES DANS LE SUD DE LA FRANCE
}

\author{
Olivier Lemercier, Yaramila Tchérémissinoff
}

\section{GÉOGRAPHIE, GULTURES ET SÉPULTURES}

Le sud de la France défini ici s'étend du CentreOuest sur la façade atlantique au Midi méditerranéen jusqu'au massif alpin, et inclut l'Auvergne, la Bourgogne orientale et le Jura. Il s'agit d'une vaste région ouverte à la fois sur l'Atlantique et la Méditerranée, offrant des contextes topographiques, climatiques et environnementaux très différents. À cette diversité naturelle correspond, à la fin du Néolithique, une variété d'entités archéologiques traduisant probablement une diversité de systèmes socio-économiques et symboliques, ainsi qu'une pluralité de relations ou d'influx culturels.

Dans cette grande moitié sud de la France, le III $^{\mathrm{e}}$ millénaire avant notre ère est marqué par un grand nombre d'entités culturelles, plus ou moins bien connues selon les régions. Si celles-ci reflètent sans doute une histoire et un état de la recherche liés à quelques archéologues très actifs du $\mathrm{Xx}^{\mathrm{e}}$ siècle et à une tradition de l'archéologie régionaliste, la diversité des styles céramiques par exemple ne saurait être remise en cause et doit bien recouvrir une certaine réalité préhistorique. Au milieu du $\mathrm{III}^{\mathrm{e}}$ millénaire avant notre ère, période où l'on s'accorde à faire apparaître les premiers éléments campaniformes en France, malgré l'indigence des datations absolues disponibles, la moitié sud de la
France semble cependant connaître une ambiance culturelle générale que l'on définit en terme de «rayonnement» de deux grandes entités culturelles : le groupe de Fontbouïsse dans les régions méditerranéennes et le groupe d'Artenac sur la façade atlantique et ses marges. Une multitude de faciès micro-régionaux partagent de nombreux traits de la culture matérielle de ces deux groupes alors que d'autres entités, marginales, en subissent sans doute l'influence elle-même sensible à travers l'évolution de la culture matérielle.

Sur la façade atlantique, c'est la culture d'Artenac qui, pendant le $\mathrm{III}^{\mathrm{e}}$ millénaire, semble réunir des régions de cultures diverses dans les siècles précédents. La fin du Néolithique en Auvergne demeure méconnue et mal caractérisée d'un point de vue chronoculturel. Dans les régions méditerranéennes, le rayonnement du groupe de Fontbouïsse se perçoit grâce à l'apparition de certaines morphologies céramiques dans les cultures situées aux marges du Languedoc oriental, le Vérazien récent en Languedoc occidental et central, le groupe des Treilles sur les Causses, le groupe Rhône-Ouvèze en Provence (Lemercier, 2007). Cette influence fontbuxienne s'étend sans doute aussi au-delà dans le Néolithique final rhodano-alpin et jusque dans le bassin de la Saône où l'entité Saône-Rhône (en attente d'une redé- 
finition) présente des traits méditerranéens évidents et probablement fontbuxiens.

Le sud de la France au milieu du III $^{\mathrm{e}}$ millénaire avant notre ère est donc marqué tout à la fois par une diversité culturelle mais aussi une sorte d'ambiance commune. Le développement conjoint des études monographiques de sites et des études spécialisées de matériaux et de techniques concernant la fin du Néolithique a, par ailleurs, clairement mis en évidence une contradiction des observations portant, d'une part, sur le recentrage des territoires d'approvisionnement des groupes humains autour de leur habitat, le développement des marqueurs identitaires et de certaines formes de monumentalité et, d'autre part, sur l'existence et peut-être le développement de relations à longue distance qui se traduisent par des mouvements d'échange et de diffusion que l'on peut observer aussi bien pour certaines matières siliceuses que pour les objets en métal.

Le domaine funéraire renvoie à cette même dualité entre une pratique funéraire largement partagée sur l'ensemble du sud de la France, qui est celle de la sépulture collective, et une extrême variété funéraire dans les types de tombes et l'architecture des monuments liés, semble-t-il, à des traditions culturelles fortes ainsi qu'à des configurations ou opportunités environnementales. Selon les régions et les cultures, les sépultures collectives sont implantées dans des grottes naturelles ou artificielles, des dolmens d'architecture variée, comme des allées couvertes. Et cette pratique dominante de la sépulture collective ne semble pas avoir fait totalement disparaître la sépulture individuelle qui demeure présente dans certaines régions et certaines cultures.

C'est dans ce contexte culturel et funéraire varié et complexe que le Campaniforme va apparaître et se développer dans la moitié sud de la France.

\section{LES SÉPULTURES DU CAMPANIFORME AU BRONZE ANCIEN I DANS LE SUD DE LA FRANCE}

\section{LA VARIÉTÉ DU CORPUS FUNÉRAIRE}

\section{Du Centre-Ouest aux Pyrénées}

Pour le sud de la façade atlantique, les informations funéraires sont très lacunaires. Les indices campanifor- mes proviennent de sépultures fréquentées sur de longues durées, grottes et dolmens (Boulestin, Gomez de Soto, 2005, p. 66).

Les exemples les mieux caractérisés sont plutôt septentrionaux. Il s'agit de la sépulture de La Folie, à Poitiers dans le département de la Vienne (Y. Tchérémissinoff et al., ce volume, chap. I, p. 11), qui renvoie aux expressions funéraires du standard $A O O$ de la basse vallée du Rhin et de l'enclos des Terriers à Avrillé, en Vendée (Bénéteau et al., 1992), une sépulture ou structure péri-sépulcrale, livrant un mobilier très homogène qui relève d'un Campaniforme régional. C'est à cet horizon que se rattache aussi le tumulus de Jard-surMer, en Vendée (Joussaume, 1968), malheureusement très érodé. Ces trois exemples suggèrent une possible filiation entre les sépultures à enclos du Bronze ancien et les sépultures du standard rhénan, au sein de l'aire de diffusion campaniforme très active qu'est la façade atlantique (Salanova, 2000).

Dans ce cadre, on évoquera l'enclos du Fief-du-Chail à Port-d'Envaux, en Charente-Maritime (Ernaux et al., 1999). Il s'agit d'une structure de grand diamètre (14 m), qui ne possédait pas (plus) de structure(s) centrale(s) mais a livré une sépulture dans son fossé; ce qui est courant dans ce type de sépultures au Bronze ancien à plus large échelle. On se doit également d'évoquer dans le courant des sépultures à enclos les deux sépultures de Cram-Chaban, en Charente-Maritime (Barbier, 1998; Barbier et al., 1998). Ces deux sépultures individuelles ont été aménagées au sein d'un fossé d'enclos simple pour l'une, et de trois fossés concentriques pour l'autre. Leur architecture, qui consiste en un coffre périssable placé dans une fosse large et bloqué par un empierrement conséquent, présente une forte parenté avec les sépultures des ensembles auvergnats (cf. infra).

Dans les Pyrénées occidentales et centrales, les expressions funéraires campaniformes sont issues d'ensembles collectifs (Guilaine et al., 1989, p. 148) ou, tout au moins, de sépultures témoignant de longues périodes de fréquentation continues ou ponctuelles. On relève toutes sortes de contextes (Janin, 2001). C'est d'une certaine façon aussi le cas des grands ensembles tumulaires, dont l'attribution à l'âge du Fer prédomine, mais au sein desquels les vestiges fini-néolithiques, bien que ténus, apparaissent constants et évoquent des « fondations» chalcolithiques.

Quant aux tombes associées au standard céramique du Campaniforme, on peut surtout citer les tumulus détruits de La Halliade à Bartrès, dans les Hautes-Pyré- 
nées (Piette, 1881, p. 44, pl. XVII, fig. 3 et 5 ) et aussi mentionner la découverte plus récente d'un tesson portant des décors de type maritime, de signification délicate, dans le fossé d'enclos d'un tumulus à AvezacPrat-Lahitte, également dans les Hautes-Pyrénées (Tchérémissinoff et al., 2008). Hors du tumulus déjà signalé de La Halliade, les formes funéraires bien caractérisées se rapportent plutôt au Bronze ancien. C'est le cas du groupe sud-aquitain du Pont-Long, défini par F. Marembert (2000), qui relève cependant d'une ascendance campaniforme bien marquée.

\section{Du Toulousain au Languedoc central}

Dans le Toulousain, le Campaniforme semble essentiellement connu dans des contextes d'habitat ou indéterminés.

Dans l'Ariège, seuls deux dolmens livrant du mobilier campaniforme peuvent être mentionnés (Treinen, 1970).

Dans l'Aude, les sépultures ayant livré des objets campaniformes sont essentiellement collectives. On recense un peu plus d'une trentaine de tombes ou groupes de tombes contenant du mobilier campaniforme, qui se répartissent équitablement entre sépultures en cavité et sépultures mégalithiques. Il s'agit systématiquement de sépultures collectives utilisées par les groupes antérieurs du Néolithique final (Guilaine, 1967; Guilaine et al., 1994; Ambert, 2003). De rares sépultures individuelles relèvent du Bronze ancien. On peut citer la sépulture 2014 de Limoux, sans mobilier, qui constitue peut-être l'une des rares tombes «pleine-terre» du corpus (Tchérémissinoff $e t$ $a l ., 2010)$. Une sépulture d'enfant en fosse domestique (silo) est également signalée sur le site du Roc-d'enGabit à Carcassonne, où une seconde sépulture d'enfant de même type, mais non datée, pourrait être rattachée à la même occupation (Vaquer et al., 2004). Les autres exemples se rapportent plutôt à un Bronze ancien évolué.

Il en est de même pour les Pyrénées-Orientales où les sépultures livrant du mobilier campaniforme sont moins nombreuses mais se répartissent entre cinq sépultures en cavité connues et six monuments mégalithiques (Claustre, Mazière, 1998 ; Janin, 2001). Certains petits dolmens, comme celui de l'Oliva-d'en-David à Salses, pourraient relever d'une édification tardive dans le contexte général. Son coffre de plan trapézoïdal (semi-enterré et sans accès permanent), qui a reçu au moins cinq défunts, relève même sans doute d'un Bronze ancien assez évolué (Bocquenet, Valentin, 1995 ; Claustre, 1997).

Dans l'Hérault, où dix-huit dolmens ont livré du mobilier campaniforme (Montjardin, 1996), deux possibles sépultures en fosse sont aussi signalées. Il s'agit de la sépulture du Chemin Dupeyne (Alignan-du-Vent), qui aurait contenu les restes d'un unique individu associé à de la céramique du Campaniforme ancien, de style mixte (Ambert, 2003; Espérou, à paraître), ainsi que la structure 39 du site de Richter (Montpellier), où un fémur humain était associé à un gobelet campaniforme de la phase ancienne là aussi et de style mixte (information L. Jallot).

Concernant le nord du Languedoc, les Causses et les contreforts du Massif central, les contextes antérieurs sont pareillement réoccupés. B. Pajot (1999, p. 143-172) remarque cependant une diminution du nombre de défunts présents dans les dolmens (préalablement vidangés?) au Bronze ancien. Pour ce secteur géographique, comme pour la Provence (cf. infra), la discussion portant sur de possibles édifications de petits monuments durant le Campaniforme et le Bronze ancien n'est pas close. Ainsi est-il probable que sous le tumulus Des Gardes à Montjaux (Aveyron), un petit coffre à accès vertical comportant une sépulture individuelle - dont les offrandes renvoient de manière homogène au Bronze ancien - a été sans doute édifié durant cette dernière période (Audibert, Dalord, 1959; Maury, 1967; Clottes, Costantini, 1976 ; Pajot, 1983 ; ThauvinBoulestin, 1998).

On observe par ailleurs «l'extraction» des unités d'inhumation autour des monuments, par exemple à Château-Bû (Causses du Quercy) où deux sépultures individuelles du Bronze ancien ont été installées juste en arrière du chevet d'un dolmen (Thauvin-Boulestin, 2000).

\section{La Provence et le Bassin rhodanien}

Dans le Bassin rhodanien, de la région lyonnaise à la Provence et au Languedoc oriental, une centaine de sépultures a fourni des mobiliers campaniformes. Il s'agit en grande majorité de sépultures collectives en cavité ou en monument mégalithique et parfois en hypogées qui correspondent aux traditions funéraires antérieures. La plupart de ces sépultures - même les sépultures construites - contiennent des objets strictement antérieurs au Campaniforme et témoignent 
d'une réutilisation ou d'une poursuite de l'utilisation de monuments édifiés plus tôt dans le Néolithique final (Lemercier, 2004).

Les cavités sont très largement utilisées, qu'il s'agisse d'abris peu profonds ou de véritables grottes. Certaines semblent avoir fait l'objet d'aménagements comme la grotte de Cost, à Buis-les-Baronnies dans la Drôme (Vignard, 1961, p. 394-396). Tous les types de monuments mégalithiques ont livré des éléments campaniformes; il en est ainsi des petits dolmens d'Ardèche et du nord du Gard, des dolmens alpins comme celui du Villard (Le Lauzet-Ubaye, Alpes-de-Haute-Provence), des dolmens à petite chambre carrée de Provence orientale et des dolmens à longue chambre de Provence occidentale, dans la basse vallée du Rhône, jusqu'aux rares monuments septentrionaux comme la tombe de Vernas en Isère (Bocquet, 1976). Les grands monuments (dolmens et allées couvertes) de Fontvieille (Bouches-du-Rhône) ont aussi livré des éléments campaniformes (Lemercier, 2004), ainsi que certains hypogées comme celui du Perpétairi à Mollans-sur-Ouvèze, dans la Drôme (Courtin, 1961c), peut-être également celui des Huguenots à Mirabel-aux-Baronnies (Drôme) détruit anciennement et l'abri Coutelier à Grillon dans le Vaucluse, dont la majorité du mobilier céramique pourrait relever de la phase initiale du Bronze ancien (Vital [dir.], étude en cours).

Finalement, les tombes exclusivement campaniformes sont très rares, qu'il s'agisse de sépultures collectives, plurielles ou individuelles. La sépulture individuelle d'adulte de La Fare, à Forcalquier (Alpesde-Haute-Provence), pourrait être considérée comme telle en fonction de la pratique funéraire observée, mais elle présente des particularités évidentes (O. Lemercier et al., ce volume, chap. XI, p. 145). Les autres sépultures en fosse connues pour le Campaniforme stricto-sensu sont des sépultures d'enfants en très bas âge, telles que celle de la grotte Murée à Montagnac, dans les Alpes-de-Haute-Provence (J. Courtin et al., ce volume, chap. XII, p. 161) et celle sur le site des Barres à Eyguières, dans les Bouches-du-Rhône (Mahieu, 1992, p. 76 et fig. 4). L'ensemble funéraire des Juilléras à Mondragon (Vaucluse) est attribuable à la phase tardive du Campaniforme et présente une variété de structures funéraires (fosses, coffres), en même temps qu'une pratique très codifiée (Lemercier et al., 2002 ; Lemercier, Tchérémissinoff, 2002 ; Lemercier, 2004, p. 420).

Quelques structures différentes sont aussi mentionnées. Il s'agit de la tombe en blocs ou tholos 2 de Sainte-Anne ou de Caillassou à Saint-Vallier-de-Thiey
(Alpes-Maritimes), qui a livré deux individus dans une petite chambre rectangulaire composée de blocs et incluse dans un tumulus d'une dizaine de mètres de diamètre (Bottin, 1885; Courtin, 1974), ou de petits coffres comme celui des Puades I à Saint-Cézaire, également dans les Alpes-Maritimes (Gagnière, 1972). Des structures tumulaires sont aussi connues dans divers secteurs de cette région, mais dont les fouilles anciennes ou la présence de structurations de dalles et de blocs ne permettent pas toujours d'affirmer qu'il s'agit réellement de tumulus ou de monuments mégalithiques ruinés. C'est le cas de plusieurs monuments dans les Alpes-Maritimes: le tumulus 1 du Plan-desNoves à Vence (Blanc, 1873), qui présente une chambre composée de dalles et de murets (Lemercier, 2004, fig. 75); le tumulus de la Collette à Escragnolles (Chiris, 1889), qui pourrait être une "tholos», selon J. Courtin (1974), par la présence d'une couronne de blocs; le tumulus des Passages à Saint-Vallier-de-Thiey, fouillé anciennement (Bottin, 1885), qui aurait peutêtre présenté une chambre (Gassin, 1986). Par ailleurs, en Ardèche, les tumulus du Serre d'Aurouze à Soyons (Blanc, 1958) et de Sabatas à Chomérac (Combier, 1963) présentaient tous les deux des aménagements de dalles qui limitaient le dépôt funéraire. Enfin, le tumulus de la Grande Bastide au Plan-d'Aups, dans le Var (Courtin, 1974), a été détruit sans observation préalable. Les seuls monuments fouillés qui se présenteraient comme de réels tumulus sont celui de Peirachier à Saint-Vallier-de-Thiey, attribué au Campaniforme par G. Sauzade (1998) sans mention de mobilier spécifique, et le tumulus du Gendarme au Plan-d'Aups (Courtin, 1974).

Il convient de signaler dans la zone intermédiaire, entre le Bassin rhodanien et l'Auvergne, la découverte très récente sur le site de la carrière Cemex à Chambéon (Loire) d'une fosse de petites dimensions $(1,20 \mathrm{~m}$ $\times 0,70 \mathrm{~m})$ qui a livré deux gobelets campaniformes dont un décoré dans le style incisé et estampé de la phase récente. Malgré l'absence de restes humains, dans un sédiment acide, il s'agit très probablement des vestiges d'une sépulture individuelle (études en cours, C. Vermeulen, Inrap).

\section{De l'Auvergne Au Jura}

Deux secteurs géographiques, outre la façade atlantique déjà évoquée, permettent de faire le lien avec des pratiques funéraires septentrionales ou orientales: 
l'Auvergne et le bassin de la Saône (Bourgogne orientale et Jura).

Concernant l'Auvergne, la seule tombe pouvant se rattacher à une expression standardisée est la sépulture 2 de La Gravière Peer II, à Riom dans le Puy-deDôme (Loison, 2003, p. 55-57). Il s'agit d'une sépulture masculine, dont le mobilier se compose d'un brassard d'archer et de deux armatures en silex pédonculées à ailerons équarris. L'architecture implique un coffre putrescible, installé dans une large fosse rectangulaire. Le défunt a été placé dans une position fléchie sur le côté gauche, dans une orientation sud-nord, une orientation qui ne domine pas au Bronze ancien dans cette région. Les autres tombes intègrent des expressions plus évoluées, régionalisées ou épicampaniformes, comme celle de Chazal à Pont-du-Château, Puy-deDôme (Loison, 2003, p. 51-54) ou sur le site du Brezet à Clermont-Ferrand (Blaizot, Vernet, 2004). Il s'agit de deux sépultures en fosses domestiques, concernant trois défunts. Elles ne livrent pas d'offrandes explicites, mais révèlent des gestes complexes. Ainsi, pour la sépulture du Brezet, un bloc crânio-facial a été prélevé sur le premier dépôt primaire et un dépôt secondaire de trois crânes, dont celui prélevé, a été réalisé plus haut dans le comblement. À Pont-du-Château, un troisième défunt, un jeune enfant, a été installé suite à la réouverture d'un espace dévolu à un dépôt double. Ces gestes présentent de fortes parentés avec des procédures couramment observées dans les sépultures «closes»du Bronze ancien (cf. infra). L'Auvergne constitue également un cas un peu à part dans un large Sud, et même à l'échelle de notre territoire, dans la mesure où cette région a livré les seuls ensembles assimilables à des nécropoles pour le Bronze ancien. Comme pour le sud de l'Allemagne et l'Europe centrale d'une manière générale, domaines auxquels les expressions renvoient très explicitement (Loison, 2003, p. 119), ces ensembles paraissent très étendus et s'organisent en cellules d'environ cinq à dix tombes. Cette configuration n'est pas sans rappeler le site normand de Bernières-sur-Mer dans le Calvados (J.-Y. Noël, ce volume, chap. IV, p. 47) ou le site Lorrain de la Sente en Moselle (Lefebvre et al., 2008). Pour ces deux sites, les nécropoles semblent initiées à la fin du Campaniforme, ce qui est peut-être aussi le cas du plus important ensemble de sépultures individuelles (72 unités, 83 défunts) actuellement connu pour le Bronze ancien en France: la nécropole de Chantemerle à Gerzat dans le Puy-de-Dôme (Vermeulen, 2002; Vermeulen et al., 2002), qui s'organise autour de sépultures encloses valorisées (7) ayant livré du mobilier de tradition campaniforme ${ }^{28}$.

En Bourgogne orientale, dans le bassin de la Saône, les sites funéraires campaniformes sont pour l'essentiel des sépultures collectives antérieures, coffres mégalithiques et dolmens qui se concentrent sur les collines et plateaux qui bordent la vallée de la Saône à l'ouest. Quelques structures tumulaires comme les tumulus 2, 3 et 4 du site des Croconnets (Bouze-lès-Beaune, Saôneet-Loire) ont livré du mobilier campaniforme (Salanova, Ducreux, 2005).

Les sépultures individuelles demeurent très rares. Découvert anciennement, le tumulus 1 de Vertempierre, à Chagny en Saône-et-Loire, n'a livré qu'un unique individu associé à un gobelet et des parures probablement campaniformes (Thevenot, 1961). La sépulture fouillée en 1912, qui était implantée dans un petit tumulus, n'a cependant pas été observée du point de vue de la structure ni du dépôt funéraire.

Beaucoup plus récemment, la fouille préventive du site des Quétignières à Longvic (Côte-d'Or) a livré une sépulture individuelle dans une petite fosse quadrangulaire, associée à un gobelet très érodé mais peut-être de style ancien et quatre coquilles de bivalve percées (Salanova, Ducreux, 2005). Une sépulture présentant des éléments de parures similaires, mais sans plus de description, est mentionnée aux Bourroches à Dijon, en Côte-d'Or (Salanova, Ducreux, 2005).

Pour le Jura, les sépultures se rapportant au Campaniforme strict ne sont pas connues (ou disponibles?). Les ensembles tumulaires tels que ceux du Salinois, récemment réexaminés (Piningre, Ganard, 2004), se rapportent très majoritairement à un plein Bronze ancien. Comme pour les nécropoles auvergnates, cependant, la question d'une fondation plus ancienne, Chalcolithique ou Bronze ancien I, est latente (Piningre, Ganard, 2004, p. 214). C'est également le cas pour l'ensemble plus «méridional» partiellement fouillé du Recourbe, à Château-Gaillard dans l'Ain. L'une de ces sépultures est localisée au sein d'un grand enclos et la trame des implantations évoque un ensemble étendu, organisé sous forme de cellules. Les architectures, qui renvoient à la présence de coffres putrescibles, intègrent des dispositifs de pierres dans de larges fosses. Ce schéma correspond à celui qui domine au Bronze ancien dans les nécropoles auvergnates et probablement jurassiennes (il s'agit de fouil-

28. Dont l'étude est encore en cours. 
les anciennes). À noter le développement de positions étendues dans les ensembles du Salinois et du Mâconnais (Barthélémy, 1976), qui vont aussi dominer en Suisse occidentale au plein Bronze ancien.

\section{TRADITIONS ET TRANSFORMATIONS DES PRATIQUES FUNÉRAIRES}

\section{Une PRATIQUe dominante: le CAMPaniforme en SÉPULTURES COLLECTIVES}

Dans l'ensemble des régions concernant le sud de la France, l'usage des sépultures collectives, généralement antérieures, semble être la règle. Si l'inventaire des sépultures livrant du mobilier campaniforme demeure aujourd'hui incomplet, les régions les mieux connues montrent une proportion de sépultures collectives que l'histoire de la recherche ou les probabilités de découverte ne sauraient expliquer en totalité.

Ainsi, les décomptes des sépultures livrant du mobilier campaniforme ou attribuées au Campaniforme (toutes phases et styles confondus) indiquent, pour les départements du littoral méditerranéen (des Alpes-Maritimes aux Pyrénées-Orientales), cent trente et une sépultures dont seulement six sépultures individuelles, peut-être huit si on inclut des données anciennes ou peu fiables. Dans la même région, les sépultures campaniformes plurielles se limiteraient au cas de la tholos 2 de Sainte-Anne à Saint-Vallier-deThiey (Alpes-Maritimes). Ces données montrent en même temps que les vestiges campaniformes apparaissent dans cent vingt-deux contextes funéraires collectifs en cavité ou en monument. Les sépultures individuelles ou plurielles ne représentent donc, au mieux, que $7 \%$ des cas.

Sur la façade atlantique, les sépultures individuelles seraient au nombre de six (si l'on prend en compte des tombes attribuées sans mobilier spécifique, par datation...), auxquelles on peut ajouter une sépulture double, sur un total recensé (non exhaustif) d'une cinquantaine de sépultures entre la Loire et la Gironde. Une sépulture individuelle ayant livré une hache-marteau perforée est par ailleurs attribuée au Cordé: Les Gâts à Tancoigné, dans le Maine-et-Loire (Patte, 1953).

Dans le Sud-Ouest, des Pyrénées aux Causses, les sépultures individuelles attribuables au Campaniforme sont absentes alors qu'un minimum de vingt-cinq monuments mégalithiques livrent des vestiges de gobelets.

Dans les Alpes et la vallée du Rhône, sur un total d'une cinquantaine de tombes recensées, une quinzaine sont individuelles et deux doubles ou plurielles, mais cela en comptant chaque sépulture de la petite cellule d'inhumations des Juilléras à Mondragon (Vaucluse), car le décompte en sites funéraires réduirait ce groupe à sept cas.

En Bourgogne orientale, sur vingt-trois tombes inventoriées, trois sont des sépultures individuelles ou supposées telles.

Finalement, seule l'Auvergne ne compte qu'une seule sépulture collective mentionnée pour trois sépultures individuelles ou multiples attribuables au Campaniforme ou à la toute première phase du Bronze ancien.

Dans cette grande moitié sud et en l'état actuel des inventaires, dans lesquels nous pourrons sans doute ajouter un certain nombre de sépultures collectives mais peu de sépultures individuelles généralement mieux connues, sur les deux cent quatre-vingt-six contextes funéraires livrant des objets campaniformes, seules trente-cinq tombes individuelles et plurielles (en tenant compte de tous les cas discutables) peuvent être mentionnées, représentant au maximum $12 \%$ du corpus en nombre de tombes. En plus de ces tombes campaniformes, une dizaine de sépultures individuelles ou plurielles appartenant au Bronze ancien I (non campaniforme) peut être prise en compte.

Ce bref inventaire étant surévalué par la présence de découvertes anciennes ou hypothétiques et de tombes attribuées par leurs seules datations radiocarbone, les sépultures individuelles campaniformes pouvant être considérées comme attestées se limitent en réalité à une vingtaine de cas qui ne représenteraient que $7 \%$ du total...

Si l'usage de la sépulture collective semble représenter près de $90 \%$ des contextes funéraires actuellement connus et sans doute plus, il ne présente pas une réelle homogénéité. En effet, ces sépultures collectives sont de natures très diverses, entre sépulture en monument et sépultures en cavité naturelle ou artificielle. Les monuments mégalithiques ou tumulaires sont très largement majoritaires sur les cavités à l'échelle du sud de la France, mais les usages diffèrent d'une région à l'autre puisque si les cavités funéraires livrant du Campaniforme sont presque absentes sur la façade atlantique, dans le Sud-Ouest, en Auvergne et jusqu'en Bourgogne orientale, elles sont bien représentées sur 
le littoral méditerranéen et dans la région rhodanienne.

Les monuments mégalithiques livrant des mobiliers campaniformes relèvent aussi de très nombreux types et reflètent les groupes mégalithiques de chaque région, traduisant d'une manière générale la réutilisation ou la poursuite de l'utilisation de monuments antérieurs du Néolithique final, dans les traditions locales.

\section{UNE PRATIQUE ÉMERGENTE: LES PROGESSUS DE MORCELLEMENT DES SÉPULTURES COLLECTIVES}

Comme on l'a vu, les sépultures collectives restent largement majoritaires dans le sud de la France durant le Campaniforme, et même au-delà jusque dans le Bronze moyen, sur les Causses (Pajot, 1999; ThauvinBoulestin, 1998) ou en Provence (Lemercier et al., 2004).

Il faut cependant noter que le nombre de défunts accueillis dans ces ensembles est très variable et que les chambres livrant des informations anthropologiques restent extrêmement rares. Même lorsque les caveaux offrent une grande capacité, on sait désormais qu'ils ne concernent pas toute une collectivité, mais que les défunts retrouvés sont issus de tris (Leclerc, 2007). Par ailleurs, les grandes chambres se décomposent en différents secteurs techniques (Leclerc, 1997), mais également sociaux ou familiaux. Ces espaces peuvent être matérialisés physiquement (murettes, dalles, parois périssables), mais aussi s'organiser autour de certains défunts ou dépôts (divisions symboliques).

Même s'ils demeurent majoritaires, ces ensembles collectifs se fragmentent résolument à la fin du $\mathrm{III}^{\mathrm{e}}$ millénaire avant notre ère. Ainsi la taille des chambres, dont la construction peut être rattachée au Campaniforme ou au Bronze ancien, est plus modeste, plus fermée, et accueille un plus petit nombre de défunts (Chambon, 2003, p. 331-339): comme si les sectorisations, précédemment citées, s'extrayaient des chambres pour former des ensembles clos autonomes. Ce phénomène d'éclatement des sectorisations, qui avait déjà été modélisé pour d'autres domaines (Boujot, 1996; Chambon, 2004a et b), est bien lisible dans le Bassin rhodanien (Tchérémissinoff, 2006, p. 14, 17 et 31). C'est aussi le cas au sein des ensembles collectifs, ici les cavités, mais aussi à travers le grand nombre de sépultures plurielles (Tchérémissinoff, 2006, p. 15), qui représentent un tiers environ des manifestations «non-collectives» au Bronze ancien. À large échelle, différentes manipulations de caractère archaïsant sont par ailleurs fréquemment observées au sein des sépultures plurielles, comme on l'a vu pour la sépulture auvergnate du Brezet.

\section{LES SÉPULTURES INDIVIDUELLES DU CAMPANIFORME ET DU BRONZE ANCIEN I DANS LE SUD DE LA FRANCE}

Un recensement des découvertes connues de sépultures individuelles ou contenant un petit nombre d'individus à l'échelle du sud de la France, nous a permis de réunir quarante-cinq tombes réparties entre la Loire et le Val de Saône au nord et la Méditerranée au sud (tabl. XXIII et fig. 106).

Dans le cadre de la recherche d'occurrences qui suit, le corpus retenu concerne finalement les sépultures les plus documentées (et surtout celles publiées) et les plus fiables en terme d'attribution chronoculturelle. Bien qu'elles nous aient permis d'étendre la réflexion générale aux contextes, les sépultures nord atlantiques ne peuvent être retenues ici comme méridionales.

Ce corpus est donc majoritairement méditerranéen. Il est également très déséquilibré en nombre d'unités par rapport à la moitié nord de la France. Nous y avons également adjoint les quelques sépultures connues du Bronze ancien I, en vue d'enrichir la réflexion sur le rôle du Campaniforme dans le processus d'individualisation des espaces funéraires.

Les sépultures ainsi retenues sont au nombre de trente-trois et concernent au moins quarante-sept défunts. Sept d'entre elles, en effet, ont reçu de deux à cinq individus. Concernant l'identité des individus inhumés, nous dénombrons au moins vingt adultes, dont seuls quatre font l'objet d'une diagnose fiable ( 1 femme et 3 hommes), et vingt-trois sujets immatures de tous âges, dont deux périnataux et deux adolescents. Le déficit de détermination pour les adultes constitue évidemment un biais important pour la réflexion proposée.

Les sépultures plurielles, enfin, associent systématiquement des adultes et des enfants ou des enfants entre eux. Dans les Alpes-Maritimes, le tumulus de SainteAnne 2 constitue une exception discutable (2 adultes), dans la mesure où il s'agit probablement d'un dolmen réoccupé, à l'image du tumulus des Passages (au minimum 6 individus). 
Tabl. XXIII — Liste des sépultures individuelles et plurielles du Campaniforme au Bronze ancien I dans la moitié sud de la France.

\begin{tabular}{|c|c|c|c|c|c|c|c|c|}
\hline $\mathbf{N}^{\circ}$ & Secteur & Département & Commune & Site & Sépulture & Phasage & Datation & Bibliographie \\
\hline 1 & Centre-ouest & Vendée & Jard-sur-Mer & Le Paradis-aux-Ânes & - & récent & - & Joussaume, 1981 \\
\hline 2 & Centre-ouest & Vendée & $\begin{array}{l}\text { Saint-Martin-de- } \\
\text { Fraigneau }\end{array}$ & Boulloires & - & récent ou tardif & $\begin{array}{l}\text { Ly-7487 : } 3700 \pm 50 \mathrm{BP} \\
\text { soit } 2280-1940 \text { cal. BC }\end{array}$ & André, 1998a \\
\hline 3 & Centre-ouest & Vendée & Auzay & Les Sables-de-l'Oie & - & indéterminé & $\begin{array}{c}\text { Lyon-43 / OxA-4723: } \\
3935 \pm 55 \text { BP } \\
\text { soit } 2580-2230 \text { cal. BC }\end{array}$ & André, 1998b \\
\hline 4 & Centre-ouest & Maine-et-Loire & Tancoigné & Les Gâts & - & culture cordée? & - & Patte, 1953 \\
\hline 5 & Centre-ouest & Vienne & Aslonnes & Tumulus de Laverré & - & ancien & - & Patte, 1971 \\
\hline 6 & Centre-ouest & Vienne & Poitiers & La Folie & - & ancien & $\begin{array}{c}\text { GrA-18765: } 3835 \pm 45 \text { BP } \\
\text { soit } 2470-2140 \text { cal. BC } \\
\end{array}$ & $\begin{array}{c}\text { Tchérémissinoff et al., } \\
\text { ce volume }\end{array}$ \\
\hline 7 & Centre-ouest & Charente-Maritime & Dolus & L'Écuissière & - & indéterminé & $\begin{array}{l}\text { Ly-7469: } 3885 \pm 50 \text { BP } \\
\text { soit } 2480-2200 \text { cal. BC }\end{array}$ & \multirow{2}{*}{ Laporte, 2008} \\
\hline 8 & Centre-ouest & Charente-Maritime & Cram Chaban & Enclos & - & indéterminé & $\begin{array}{l}\text { Ly- } 8453: 3690 \pm 50 \mathrm{BP} \\
\text { soit } 2210-1930 \text { cal. } \mathrm{BC}\end{array}$ & \\
\hline 9 & Auvergne & Puy-de-Dôme & Pont-du-Château & Chazal & - & tardif? & $\begin{array}{l}\text { LYON-374 : } 4005 \pm 70 \\
\text { soit } 2900-2250 \text { cal. BC }\end{array}$ & \multirow{2}{*}{ Loison, 2003} \\
\hline 10 & Auvergne & Puy-de-Dôme & Riom & Gravière Peer II & - & récent? & $\begin{array}{l}\text { Ly-7685: } 3830 \pm 55 \mathrm{BP} \\
\text { soit } 2470-2130 \mathrm{cal} . \mathrm{BC}\end{array}$ & \\
\hline 11 & Auvergne & Puy-de-Dôme & Clermont-Ferrand & Le Brezet & - & indéterminé & $\begin{array}{l}\text { Ly-10817 : } 3995 \pm 45 \text { BP } \\
\text { soit } 2630-2340 \text { cal. BC }\end{array}$ & Blaizot et Vernet, 2004 \\
\hline 12 & Côte Méditer. & Aude & Limoux & Cépie & 2014 & BA I & $\begin{array}{l}\text { Ly-3589: } 3610 \pm 25 \text { BP } \\
\text { soit } 2030-1890 \text { cal. BC }\end{array}$ & $\begin{array}{l}\text { Tchérémissinoff et al., } \\
2010\end{array}$ \\
\hline 13 & Côte Méditer. & Aude & Carcassonne & Roc-d'en-Gabit & - & \multirow{2}{*}{ tardif } & - & Vaquer et al., 2004 \\
\hline 14 & Côte Méditer. & Aude & Carcassonne & Roc-d'en-Gabit & - & & - & Vaquer et al., 2004 \\
\hline 15 & Côte Méditer. & Hérault & Alignan-du-Vent & Chemin Dupeyne & - & ancien & $\begin{array}{c}\text { Tuc-20951: } 4060 \pm 65 \text { BP } \\
\text { soit } 2880-2460 \text { cal. BC }\end{array}$ & $\begin{array}{c}\text { Ambert, } 2003 ; \\
\text { Espérou, à paraître }\end{array}$ \\
\hline 16 & Côte Méditer. & Hérault & Montpellier & Espace Richter & - & ancien & - & information L. Jallot \\
\hline 17 & Côte Méditer. & Hérault & Quarante & Pech-Laurier & - & BAI & - & Ambert, 1982 \\
\hline 18 & Côte Méditer. & Gard & Villeneuve-lès-Avignon & Bellevue & - & BAI & - & Roudil, 1964 \\
\hline 19 & Côte Méditer. & Gard & Laudun & Colombel & - & BAI & - & Carrière, 1892 \\
\hline 20 & Côte Méditer. & Gard & Saint-Hyppolite-du-Fort & Grotte de Rascassoles & - & BAI & - & Charles, 1971 \\
\hline 21 & Côte Méditer. & Gard & Nîmes & Georges Besse II.5 & - & tardif? & $\begin{array}{c}\text { Erl-12190 : } 3832 \pm 97 \\
\text { soit } 2600-1950 \text { cal. BC }\end{array}$ & $\begin{array}{c}\text { Tchérémissinoff et al., } \\
\text { ce volume }\end{array}$ \\
\hline 22 & Côte Méditer. & Bouches-du-Rhône & Eyguières & Les Barres & - & récent? & - & Mahieu, 1992 \\
\hline 23 & Côte Méditer. & Var & Le Plan-d'Aups & Tumulus du Gendarme & - & récent & - & Courtin et Palun, 1962 \\
\hline 24 & Côte Méditer. & Alpes-Maritimes & Soint Vallier-de Thiov & Tholos 2 de Sainte-Anne & - & récent & - & Rottin 1885 \\
\hline 25 & Côte Méditer. & Alpes-Maritimes & Sami-vanter-de-mey & Tumulus des Passages & - & recent & - & DOUII, 1005 \\
\hline 26 & Alpes-Rhône & Vaucluse & Mondragon & Les Juilléras & 1 & \multirow{9}{*}{ tardif } & \multirow{9}{*}{$\begin{array}{l}\text { datation de l'occupation : } \\
\text { AA-31697: } 3635 \pm 50 \text { BP } \\
\text { soit } 2140-1880 \text { cal. BC }\end{array}$} & \multirow{9}{*}{$\begin{array}{c}\text { Lemercier, 2002; } \\
\text { Tchérémissinoff, } 2006\end{array}$} \\
\hline 27 & Alpes-Rhône & Vaucluse & Mondragon & Les Juilléras & 6 & & & \\
\hline 28 & Alpes-Rhône & Vaucluse & Mondragon & Les Juilléras & $8 \mathrm{c}$ & & & \\
\hline 29 & Alpes-Rhône & Vaucluse & Mondragon & Les Juilléras & 21 & & & \\
\hline 30 & Alpes-Rhône & Vaucluse & Mondragon & Les Juilléras & 22 & & & \\
\hline 31 & Alpes-Rhône & Vaucluse & Mondragon & Les Juilléras & 24 & & & \\
\hline 32 & Alpes-Rhône & Vaucluse & Mondragon & Les Juilléras & 25 & & & \\
\hline 33 & Alpes-Rhône & Vaucluse & Mondragon & Les Juilléras & 27 & & & \\
\hline 34 & Alpes-Rhône & Vaucluse & Mondragon & Les Juilléras & K4-14 & & & \\
\hline 35 & Alpes-Rhône & Drôme & Chabrillan & Saint-Martin 3 & 25 & \multirow{3}{*}{ BA I } & - & \multirow{3}{*}{$\begin{array}{l}\text { Blaizot, Rimbault, } \\
2005\end{array}$} \\
\hline 36 & Alpes-Rhône & Drôme & Chabrillan & Saint-Martin 3 & 128 & & \begin{tabular}{|c|} 
LYON-1482: $3570 \pm 50 \mathrm{BP}$ \\
soit $2040-1750$ cal. BC \\
\end{tabular} & \\
\hline 37 & Alpes-Rhône & Drôme & Chabrillan & Saint-Martin 3 & 130 & & $\begin{array}{c}\text { LYON-1483 : } 3560 \pm 50 \mathrm{BP} \\
\text { soit } 2030-1750 \text { cal. BC }\end{array}$ & \\
\hline 38 & Alpes-Rhône & Vaucluse & Gigondas & Les Gouberts & - & BA I & & Sauzade, Vital, 2000 \\
\hline 39 & Alpes-Rhône & $\begin{array}{l}\text { Alpes-de-Haute- } \\
\text { Provence }\end{array}$ & Forcalquier & La Fare & - & ancien & $\begin{array}{c}\text { GrA-22988 : } 3895 \pm 40 \text { BP } \\
\text { soit } 2480-2200 \text { cal. BC }\end{array}$ & $\begin{array}{l}\text { Lemercier et al., } \\
\text { ce volume }\end{array}$ \\
\hline 40 & Alpes-Rhône & $\begin{array}{l}\text { Alpes-de-Haute- } \\
\text { Provence }\end{array}$ & Montpezat & Grotte Murée & - & récent & - & $\begin{array}{l}\text { Courtin et al., } \\
\text { ce volume }\end{array}$ \\
\hline 41 & Alpes-Rhône & Loire & Chambéon & La Carrière Cemex & - & récent & - & $\begin{array}{l}\text { information } \\
\text { C. Vermeulen }\end{array}$ \\
\hline 42 & Alpes-Rhône & Ardèche & Soyons & $\begin{array}{l}\text { Tumulus du } \\
\text { Serre d'Aurouze }\end{array}$ & - & récent & - & $\begin{array}{l}\text { Arnal, Blanc, } 1959 ; \\
\text { Blanc, } 1958\end{array}$ \\
\hline 43 & Bourgogne & Saône-et-Loire & Chagny & $\begin{array}{c}\text { Tumulus de } \\
\text { Vertempierre }\end{array}$ & - & récent & - & Thevenot, 1961 \\
\hline 44 & Bourgogne & Côte-d'Or & Longvic & Les Quétignières & - & $\begin{array}{l}\text { indéterminé } \\
\text { ancien? }\end{array}$ & $\begin{array}{c}\text { Lyon-2065(Poz) : } 4030 \pm 35 \\
\text { soit } 2840-2460 \text { cal. BC }\end{array}$ & $\begin{array}{c}\text { Salanova et Ducreux, } \\
2005\end{array}$ \\
\hline 45 & Bourgogne & Côte-d'Or & Dijon & Les Bourroches & - & BAI & - & Millotte, 1964 \\
\hline
\end{tabular}




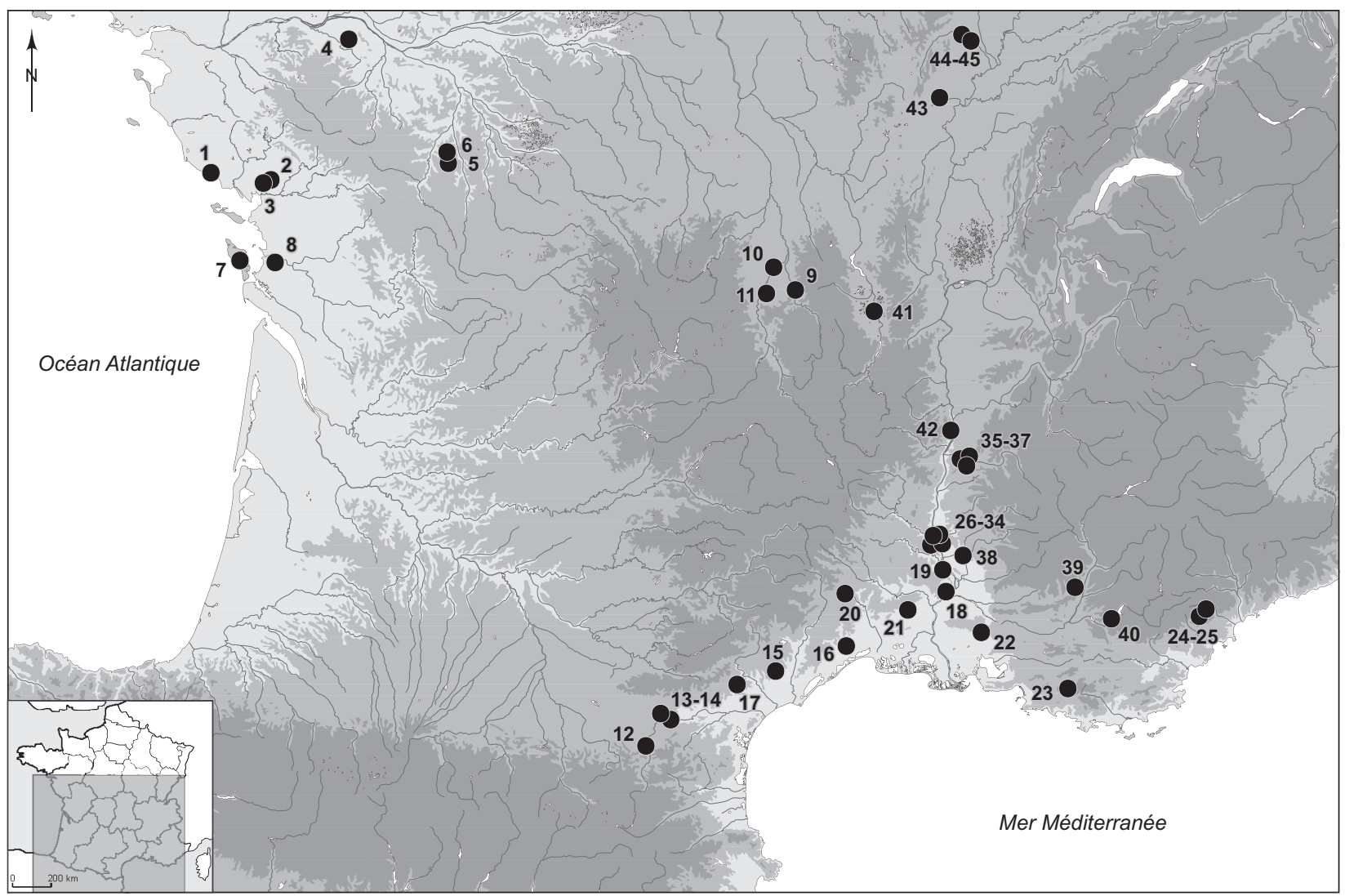

Fig. 106 - Carte de répartition des sépultures individuelles et plurielles du Campaniforme et du Bronze ancien I dans la moitié sud de la France (les numéros renvoient au tabl. XXIII).

\section{CONTEXTES DES SÉPULTURES}

\section{SÉPULTURES EN HABITAT}

Presque sans surprise, se calquant également sur des schémas plus anciens, les sépultures en fosse se retrouvent majoritairement au sein des contextes domestiques des basses terrasses alluviales (La Gravière Peer II en Auvergne et Chabrillan en Rhône-Alpes). Concernant les contextes péridomestiques, une petite catégorie nous intéresse particulièrement: celle des tombes architecturées, généralement « isolées ».

\section{SÉPULTURES ISOLÉES}

Pour le Campaniforme ancien, on pense évidemment à la sépulture de La Fare, à Forcalquier (O. Lemer- cier et al., ce volume, chap. XI, p. 145). Elle mêle originalement des caractères antérieurs (hypogéiques et mégalithiques) en une composition nouvelle, qui n'est peut-être pas sans évoquer les manifestations «à tumulus » du standard européen.

C'est également le cas de la sépulture en coffre du site G. Besse II-5, à Nîmes dans le Gard (Y. Tchérémissinoff et al., ce volume, chap. XIII, p. 167) - une sépulture à fort investissement, «isolée» au sein d'une occupation subcontemporaine - et de la tombe de La Gravière Peer II à Riom (Puy-de-Dôme) qui présente des traits campaniformes fortement marqués.

Ces trois sépultures masculines soulèvent clairement le problème des statuts individuels associés à certains marqueurs campaniformes caractéristiques, mais certainement pas avec autant d'acuité que celle de la grotte Murée, dans les Alpes-de-Haute-Provence (J. Courtin et al., ce volume, chap. XII, p. 161). Cette sépulture, qui est également isolée au sein d'un habi- 
tat, est nettement valorisée en terme de mobilier d'accompagnement et d'architecture, mais elle concerne ici un individu décédé en période périna- tale. Il s'agit d'une évocation, nous semble-t-il, très explicite en faveur de l'héritabilité des statuts individuels valorisés.

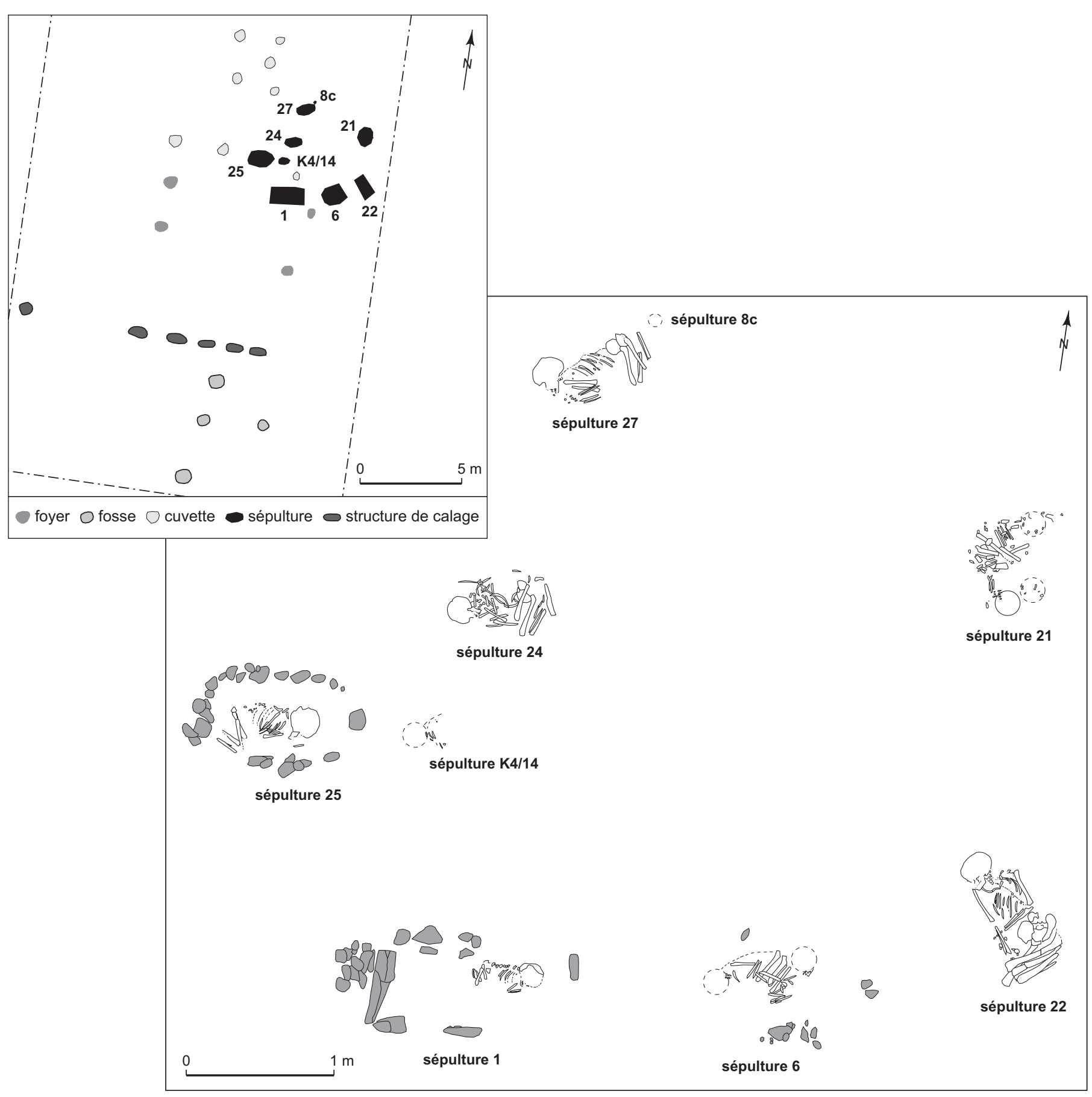

Fig. 107 - Plan de la cellule d'inhumations des Juilléras (Vaucluse), Campaniforme tardif (fouilles, relevés, DAO: O. Lemercier et Y. Tchérémissinoff). 


\section{NÉCROPOLES ET GELLULES D’INHUMATIONS}

Les contextes ne sont pas toujours bien connus, par exemple en ce qui concerne les fameux coffres de la vallée du Rhône. Un contexte funéraire peut être envisagé pour le site des Juilléras, dans le Vaucluse (fig. 107), sur le mode dominant des nécropoles en cellules dispersées observables à une large échelle pour le Bronze ancien. Dans ce cas précis, l'habitat n'est certainement pas très éloigné. Pour les tombes d'ascendances mégalithiques, en Provence par exemple (tumulus du Gendarme et de SainteAnne 2), le contexte funéraire est naturellement privilégié au regard des schémas antérieurs. C'est le cas plus généralement pour les zones de moyenne montagne (Causses du Languedoc, Pyrénées), où les ascendances chalcolithiques dominent.

\section{LES ARCHITECTURES}

\section{LES SÉPULTURES EN FOSSE}

Comme précédemment évoqué, les sépultures en fosse intègrent surtout les habitats. Il peut s'agir de fosses domestiques détournées (Chabrillan et Le Brezet), mais les espaces sont majoritairement protégés, les dépôts faisant l'objet d'une architecture plus ou moins sommaire.

\section{LES SÉPULTURES EN COFFRE}

La première catégorie des coffres concerne les compositions lithiques, d'ascendance chalcolithique et méditerranéenne. Certains exemples s'apparentent même à des dolmens (tumulus du Serre d'Aurouze, en Ardèche), parfois occultés sous le terme folklorique de «tombes à tholos» (évoquant les tombes princières à encorbellement du monde égéen), comme pour l'ensemble de Saint-Vallier-de-Thiey dans les Alpes-Maritimes, par exemple. Cette catégorie comporte généralement une signalisation de type tertre, ce qui est aussi le cas des petits coffres fermés et enterrés (à accès verticaux): une catégorie évolutive spécifique du tout début de l'âge du Bronze (G. Besse II-5, Les Gouberts, Pech Laurier et Bellevue).

\section{LES SÉPULTURES SOUS TERTRE OU CAIRNS ET LES AUTRES SYSTÈMES DE SIGNALISATION}

Concernant les signalisations empierrées (tertres ou cairns), une seule tombe en fosse domestique semble en relever: celle du site de Chazal dans le Puy-de-Dôme. Les sépultures de La Fare et de la grotte Murée (recouverte d'un tertre mixte de petites lauzes et de tessons) nous semblent, en effet, trop particulières en terme d'investissement pour intégrer la catégorie des sépultures en fosses signalées. Elles se rapprochent davantage des manifestations ostentatoires périmégalithiques.

Les tumulus sédimentés sont plus complexes à mettre en évidence car, dans des contextes érosifs, ils ne sont pas trahis par la position secondaire de leurs composantes. Dans un cas (sépulture 25 des Juilléras), la présence d'une couronne de galets plaide en faveur de l'entourage d'une levée de terre. Concernant les signalisations un peu originales, on peut mentionner le bloc d'effondrement naturel contre lequel est implantée la sépulture de la grotte Murée, qui participe à sa signalisation et en constitue même le trait mégalithique.

\section{LES MILIEUX DE DÉCOMPOSITION}

À de très rares exceptions (une sépulture en fosse à Saint-Martin), les espaces de dépôts sont protégés. Les systèmes impliquent des protections plutôt rigides et larges, les contraintes étant généralement absentes sur les squelettes et les colmatages clairement différés.

\section{LES PRATIQUES FUNÉRAIRES}

\section{Positions ET ORIENTATIONS DES DÉFunts}

Toutes les positions sont fléchies. Elles sont rarement initialement hypercontractées, dans la mesure où les espaces sont peu contraignants. Conformément $\mathrm{au}$ «modèle» du standard, les positions sur le côté gauche semblent plutôt dévolues aux hommes (La Gravière Peer II, La Fare, G. Besse II-5), mais les individus immatures et les sujets indéterminés dominent très nettement dans notre petit corpus.

Concernant les orientations, les axes est-ouest et ouest-est représentent environ deux tiers du corpus, par rapport aux axes sud-nord et nord-sud. Cette der- 
nière "variante", si elle concerne la sépulture de La Fare, n'est cependant pas propre aux sépultures de la phase I, puisqu'on la retrouve dans tout le Campaniforme.

Les inversions d'axes étant par ailleurs très équilibrées, on peut supposer qu'elles sont habituellement calquées sur le sexe du défunt, mais il n'est pas possible de le vérifier.

\section{LES SÉPULTURES PLURIELLES}

Les sépultures plurielles (de 2 à 5 défunts) représentent environ un tiers du corpus. Concernant chaque fois au moins un individu immature, elles intègrent tous les contextes d'implantations (habitats, funéraires ou intermédiaires) et tous les modèles architecturaux reconnus. Il s'agit sans doute d'un trait archaïsant, qui demeure durant tout le Bronze ancien et dans lequel s'inscrivent aussi probablement différentes manipulations post-dépositionnelles. Le cas de la sépulture des Quétignières à Longvic (Côte-d'Or) est un peu particulier, car il associe une sépulture d'adulte à une réduction (correspondant à un adulte jeune) implantée à ses pieds et qui lui serait légèrement postérieure (Salanova, Ducreux, 2005 ; informations R. Labeaune). Cette configuration pourrait trouver des comparaisons au nord-est, en Moselle, sur les sites de La Sente à Mondelange (Lefebvre et al., 2008), de Chèvres-Haies à Pouilly et de Trémery (A. Lefebvre et J. Franck, ce volume, chap. VIII, p. 97).

\section{LES MANIPULATIONS}

Bien que les espaces individualisés aient une nette tendance à se fermer au Campaniforme/Bronze ancien (coffres enterrés sans accès permanents, fosses closes, superstructures de signalisation), on observe de nombreuses ré-ouvertures accompagnées de gestes dont l'intention demeure énigmatique. C'est le cas du prélèvement associé à des dépôts secondaires dans la sépulture du Brezet, de la ponction du bloc crâniofacial de la sépulture du site G. Besse II-5 et des remaniements de la tombe de Bellevue. Pour ces deux derniers coffres, l'absence de tout mobilier conservé soulève la question si souvent évoquée à large échelle pour le Bronze ancien du pillage des mobiliers métalliques. Si l'on retient cette hypothèse, il faut cependant convenir, au minimum, que les gestes induisent des préoccupations collatérales aux pillages, d'ordre symbolique, parmi lesquelles la recomposition du dispositif de fermeture.

\section{LES MOBILIERS}

Les remarques concernant le mobilier archéologique peuvent se fonder sur un nombre plus important de tombes, prenant en compte des fouilles anciennes ou des contextes où les observations anthropologiques n'étaient pas possibles ou sont encore en cours, mais aussi les tombes qui ne livrent justement pas de mobilier spécifique. Le sud de la France considéré ici s'étend donc de la Loire au Val de Saône jusqu'à la Méditerranée.

Si le mobilier est très abondant dans les contextes funéraires livrant du Campaniforme, le faible nombre de sépultures individuelles connues n'offre qu'un mobilier relativement restreint.

Il existe des sépultures individuelles qui ne livrent aucun objet associé au corps inhumé, comme c'est le cas pour les sépultures individuelles et plurielles des Juilléras à Mondragon (Vaucluse), attribuées à l'Épicampaniforme par le contexte, les relations stratigraphiques et les datations, ainsi que pour les sépultures d'enfant du Roc-d'en-Gabit à Carcassonne (Vaquer et al., 2004). Les objets effectivement présents dans les sépultures campaniformes sont assez variés.

\section{LES DIFFÉRENTS TYPES D’OBJETS}

\section{La céramique}

Principal élément discriminant du Campaniforme, la céramique est assez fréquente dans les sépultures attribuées à cette culture, au moins pour les phases anciennes et récentes, car elle semble rare à absente pour les contextes épicampaniformes.

Elle est en effet présente dès les phases anciennes, avec l'exemple du gobelet de style mixte de la sépulture de La Fare à Forcalquier (Alpes-de-Haute-Provence), qui s'associe dans ce cas à deux petits gobelets attribuables à une culture locale du Néolithique final (O. Lemercier et al., ce volume, chap. XI, p. 149). En secteur méditerranéen, par exemple dans l'Hérault, la probable sépulture individuelle du Chemin Dupeyne (Alignan-du-Vent) aurait 
livré les restes de peut-être six vases, essentiellement des gobelets décorés dans le style mixte (Ambert, 2003; Espérou, à paraître). C'est aussi le cas dans la région atlantique avec la sépulture de La Folie à Poitiers (Vienne) qui a livré un unique gobelet $A O O$ (Y. Tchérémissinoff et al., ce volume, chap. I, p. 16), et dans le bassin de la Saône avec la sépulture des Quétignières à Longvic (Côte-d'Or), notamment les fragments d'un gobelet à décor linéaire pointillé qui pourrait, en l'attente d'une étude, être rattaché à cette phase (Salanova, Ducreux, 2005).

La céramique est aussi présente dans les sépultures des groupes régionaux de la phase récente. Pour le groupe rhodano-provençal, un fragment de coupe, une petite écuelle carénée et un bol non décoré pour la sépulture d'enfant de la grotte Murée dans les Alpesde-Haute-Provence (J. Courtin et al., ce volume, chap. XII, p. 169), un gobelet et un bol pour le tumulus du Serre d'Aurouze à Soyons en Ardèche, et un gobelet pour le tumulus du Gendarme au Plan-d'Aups dans le Var. La sépulture double du tholos 2 de Sainte-Anne à Saint-Vallier-de-Thiey (Alpes-Maritimes) a livré les restes de quatre vases, dont trois gobelets et une forme non restituable, ainsi que la sépulture plurielle du tumulus des Passages (également à Saint-Vallier-de-Thiey) a livré deux vases dont un gobelet décoré (Gassin, 1986). Sur la façade atlantique, la sépulture du Paradis aux Ânes (Jard-sur-Mer, en Vendée) a livré trois gobelets (Joussaume, 1981).

En remontant l'axe Rhône-Saône, la structure du site de la carrière Cemex à Chambéon (Loire), que nous proposons d'interpréter comme une probable sépulture individuelle, a livré deux gobelets dont un décoré. La sépulture de Vertempierre à Chagny, en Saône-et-Loire (Thevenot, 1961) a fourni un gobelet un peu atypique au décor pointillé qui pourrait relever d'une phase plutôt récente.

La céramique est finalement présente dans une unique sépulture plurielle attribuable à la phase épicampaniforme sur le site de Chazal, à Pont-du-Château dans le Puy-de-Dôme (Loison, 2003), mais à l'état de fragments ne correspondant pas obligatoirement au dépôt d'un vase.

\section{Les outillages et les armes}

Ils sont présents, sans être systématiques ni nombreux. Les poignards en cuivre sont connus dans la sépulture de La Fare (Forcalquier), dans celle du tumulus du Serre d'Aurouze (Soyons) et dans la tombe double des Boulloires à Saint-Martin-de-Fraigneau, en Vendée (André, 1998 ; Laporte, 2001, 2008), mais aussi dans des tombes du Bronze ancien I, avec la tombe du Colombel à Laudun (Gard).

Les lames et outillages lithiques sont présents dans la tombe de La Folie (une lame et un microdenticulé en silex du Grand-Pressigny), dans la tholos 2 de SainteAnne (deux lames en silex), dans la tombe d'enfant de la grotte Murée (deux lames en silex), dans le tumulus des Passages (une lame en silex) et éventuellement dans la tombe des Quétignières (un éclat de silex).

Les éléments liés à l'archerie sont aussi connus dans le tumulus du Gendarme, au Plan-d'Aups dans le Var (une armature foliacée), dans le tumulus du Serre d'Aurouze en Ardèche (un fragment d'armature biface), dans la tombe de La Gravière Peer II à Riom, dans le Puy-de-Dôme (une armature à pédoncule et ailerons équarris et un brassard d'archer) et dans la tombe double des Boulloires en Vendée (un brassard d'archer). Les tombes du début de l'âge du Bronze peuvent aussi fournir ce type d'éléments comme la tombe du Colombel dans le Gard (un brassard d'archer) et celle des Gouberts (Vaucluse) avec une armature à pédoncule et ailerons en silex.

La sépulture de la grotte Murée (Alpes-de-HauteProvence) a par ailleurs livré une petite hache polie. Pour le Bronze ancien, on note aussi la présence d'alênes métalliques dans les tombes du Colombel (Gard) et de Pech Laurier (Hérault).

\section{Les parures}

Peu nombreux, les éléments de parure sont cependant d'une grande diversité. Les perles, pendeloques et éléments de colliers sont présents à La Fare (une petite perle segmentée en os), au tumulus du Serre d'Aurouze (une perle en argent d'attribution incertaine), à Chazal (perles en bobine et en olive en calcaire, en jais, en coquilles), dans la tombe du Paradis aux Ânes (une centaine de dentales), dans la tombe des Quétignières (4 coquilles de bivalve percées), dans le tumulus des Passages (coquilles, dentales et une pendeloque arciforme à perforation médiane).

Les autres éléments sont plus anecdotiques, un objet en os en forme de bobine dans la sépulture de La Fare (bouton ou labret?) ; une défense de sanglier dans le tumulus du Gendarme ; une canine de canidé perforée, une boulette d'ocre dans la tombe de la grotte Murée et deux boutons perforés en os en forme de cheville à 
renflement médian et extrémités en tampon pour la sépulture de Vertempierre.

Pour le Bronze ancien, outre des coquilles et perles pour la tombe de Pech Laurier, les épingles apparaissent avec des cas recensés dans la tombe du Colombel et dans celle des Gouberts.

\section{LES ASSEMBLAGES MOBILIERS} ET LA QUESTION DU «PACKAGE »

Les sépultures individuelles ou plurielles du Campaniforme de la moitié sud de la France livrent globalement peu de mobilier et des assemblages souvent restreints. Si les tombes vides de tous dépôts sont nombreuses, les tombes qui ne livrent qu'un seul objet ou un seul type d'objet sont minoritaires, la plupart contenant des associations d'objets.

Les assemblages céramiques eux-mêmes sont dominés par la présence des gobelets. Les tombes montrant des associations de vases de morphologie différente sont peu nombreuses; notons cependant l'exemple de la tombe du Serre d'Aurouze qui livre un gobelet et un bol, comme celui de la sépulture d'enfant de la grotte Murée qui associe un bol et une petite écuelle carénée (le grand fragment de coupe à fond plat étant plutôt un élément constitutif de l'architecture de la tombe qu'une offrande).

Il n'est pas possible de reconnaître pour le sud de la France un assemblage particulier de type "service ", associant vase de présentation, vase de service et vase de consommation, comme c'est le cas dans certaines autres régions d'Europe et particulièrement en Europe centrale.

Les associations des trois types d'objets (céramiques, armes/outils et parures) sont peu nombreuses: La Fare, la grotte Murée, le tumulus du Gendarme et éventuellement la tombe des Quétignières (si l'on considère que l'éclat de silex, qui aurait été découvert dans la main de l'individu inhumé, constitue une offrande). Les trois types d'objets sont aussi présents dans le tumulus des Passages mais s'agissant d'une tombe plurielle, l'association des différents mobiliers peut résulter de la présence de plusieurs individus. Les associations de deux types seulement se déclinent en assemblages avec céramiques et parures dans les tombes de Vertempierre, le Paradis aux Ânes et Chazal, et avec céramiques et armes/outillages dans la sépulture de La Folie et le tumulus des Passages.
Quelques sépultures ne livrent donc qu'un seul type d'objet, comme les sépultures à céramique probablement au Chemin Dupeyne et à la carrière Cemex et des sépultures à armes/outils comme celle de La Gravière Peer II et celle de Boulloires.

La détermination des relations entre type de mobilier et statut des individus se heurte à la rareté des déterminations précises (sexe/âge). Les deux sépultures de périnataux du corpus montrent des situations contrastées avec un unique fragment de céramique dont l'intentionnalité du dépôt peut être discutée pour la tombe des Barres à Eyguières (Bouches-duRhône), face à un assemblage assez riche et diversifié pour la tombe de la grotte Murée à Montagnac dans les Alpes-de-Haute-Provence. Il est notable que les trois tombes masculines d'adultes (Boulloires, La Fare et Riom) renferment des armes (éléments d'archer et poignards), alors que la seule sépulture signalée comme féminine (Les Quétignières) associe céramique, parure et un éclat de silex. Le cas de la sépulture des Boulloires est intéressant puisqu'elle livre les restes de deux individus tête-bêche qui sont attribués tous les deux à des adultes de sexe masculin et qu'un seul de ces individus était associé au mobilier: un poignard en cuivre de type occidental et un brassard d'archer.

La position du mobilier dans les tombes ne peut être envisagée que pour quelques cas. Les céramiques peuvent se trouver aux pieds des individus inhumés comme c'est le cas à La Fare et aux Quétignières, mais aussi derrière le dos du défunt comme à La Folie et dans la grotte Murée. La structure de la carrière Cemex a livré deux vases placés à deux angles d'une petite fosse. La position des armes et des outils est aussi variable. À Boulloires, le poignard et le brassard d'archer se trouvent plutôt sur la poitrine ou l'avant du corps. Le brassard se trouvait peut-être initialement en position fonctionnelle. À La Fare, le poignard se trouve derrière le crâne de l'individu. À La Folie, la lame de silex était placée avec le vase derrière le dos du défunt alors que pour la sépulture d'enfant de la grotte Murée, les lames en silex sont disposées entre les jambes. À La Gravière Peer II, l'armature de flèche se trouvait derrière le dos de l'individu inhumé alors que le brassard était localisé en avant du corps et à toute proximité de l'avant bras.

Cette variété de type de dépôt montre à la fois des objets probablement placés en position fonctionnelle et des objets exposés à côté du corps lui-même. 


\section{PERSPECTIVES ET INTERROGATIONS}

\section{APPROCHE CHRONOLOGIQUE}

Le nombre de sépultures reconnues par période s'accroît légèrement dans l'axe temporel. Ainsi, les sépultures pouvant être clairement rattachées aux expressions internationales ou anciennes du Campaniforme demeurent très rares. Celles de La Fare, du Chemin Dupeyne, de La Folie et peut-être celle des Quétignières, comme la structure de Richter sont les seuls exemples. Les datations relatives aux tombes du Chemin Dupeyne et des Quétignières sont très hautes (2880-2460 et 2840-2460 cal BC), alors que celles de La Fare et de La Folie sont au contraire très basses (2480-2200 et 2470-2140 cal BC). Si ces dates sont justes, les premières expressions funéraires du Campaniforme dans la moitié sud de la France devraient se trouver dans leur plage de recouvrement vers 2500-2450 avant notre ère.

La sépulture de La Gravière Peer II évoque des expressions standardisées, mais sa datation, comme le rare mobilier campaniforme mis au jour sur le site, la situent plutôt au sein des expressions régionalisées. C'est à cette étape que peuvent être décomptées neuf ou dix autres sépultures. Les datations des sépultures de la phase récente stricte déterminées par du mobilier spécifique sont inexistantes.

Pour les expressions tardives, treize sépultures sont recensées, mais la seule date indirecte exploitable est celle du site des Juilléras entre 2140 et 1880 cal BC. Pour la première phase du Bronze ancien, une douzaine de sépultures intègre le corpus dont trois datées entre 2040 et $1750 \mathrm{cal}$ BC. Les autres sépultures demeurent hypothétiques ou ne peuvent être précisément datées.

\section{Le Campaniforme ancien}

La sépulture de Forcalquier évoque à la fois des manifestions funéraires du Néolithique final (hypogéisme, mégalithisme), mais de manière plutôt originale, en une association rappelant peut-être les sépultures individuelles nord-orientales. Cette association particulière se traduit également à travers son mobilier: un gobelet campaniforme et deux gobelets de tradition locale, alors que la lame de poignard associée correspond elle aussi plutôt à une expression de la métallurgie régionale qu'aux types strictement campaniformes. D'une manière générale, la présence de céramique semble être un caractère récurrent pour les tombes qui pourraient être attribuées à la phase ancienne du phénomène. Les autres catégories de mobilier (armes, outillages, parures) peuvent aussi être représentées, mais la notion de «richesse»du dépôt funéraire ne peut être avancée compte tenu du faible nombre de tombes recensées.

\section{Le Campaniforme récent}

Parmi les sépultures concernées, deux sont plurielles et trois sont des coffres lithiques dallés ou multidallés d'ascendance méditerranéenne. C'est à cette période que se rattache la sépulture de la grotte Murée, et au moins quatre de ces sépultures sont signalées par des tertres ou cairns; sinon, tous les espaces sépulcraux ont été soigneusement protégés. Le mobilier funéraire du Campaniforme récent atteste une grande diversité. Si la céramique est bien présente, c'est probablement à cette phase que peut être rattaché un certain nombre de tombes ne livrant pas de céramique campaniforme alors que les armes, outillages et parures sont largement représentés.

\section{Le CAMPaniforme taRdif ET LA TRANSITION DU NÉOlithiQue À L'ÂGE DU Bronze}

Cette phase est surtout représentée par la petite nécropole ou cellule d'inhumations des Juilléras. Il s'agit majoritairement d'espaces protégés par des matériaux putrescibles rigides (coffres ou planches); le mobilier en est absent. On relève un coffre lithique d'assez grande taille, qui concerne assez curieusement un jeune enfant. C'est aussi à cette période que se rattache le coffre de Nîmes, dont la taille, le plan trapézoïdal et la position du défunt évoquent la fameuse sépulture du Bronze ancien I de Gigondas (Y. Tchérémissinoff et al., ce volume, chap. XIII, p. 174).

Pour cette phase, d'ailleurs, cinq des douze sépultures concernées sont des coffres lithiques attestant encore une forte ascendance mégalithique. Les autres sépultures sont des fosses en contexte d'habitat, ce fait étant également courant à la fin du Néolithique dans les sites étendus des vastes terrasses alluviales, tels que ceux de la région nîmoise. Ces deux traditions ren- 
voient à des manifestations et évolutions parallèles, éventuellement liées aux statuts socio-économiques des défunts.

En ce qui concerne le mobilier archéologique, c'est sa rareté qui semble caractériser les sépultures du Campaniforme tardif et du premier Bronze ancien. Si les neuf tombes des Juilléras (Vaucluse) confirment largement cette observation, les tombes du Roc-d'en-Gabit (Aude) sont, elles aussi, vides d'offrandes, situation que nous retrouvons pour sept des douze tombes ou ensembles funéraires recensés pour le Bronze ancien I. Pour le Campaniforme tardif, seule la tombe de Chazal (Puy-de-Dôme) a livré des éléments mobiliers sous la forme de parures probablement liées à l'un des individus inhumés (et sans tenir compte de la céramique visible uniquement à l'état de fragments dans le remplissage). Pour le Bronze ancien I, les rares éléments mobiliers présents prolongent les traditions antérieures avec des poignards, armatures et brassards d'archer, mais des différences sont aussi notables avec l'apparition d'épingles métalliques et la disparition quasi-totale de la céramique qui n'est mentionnée que pour la nécropole de Chantemerle (Puy-de-Dôme).

\section{LE CAMPANIFORME DANS L'ÉVOLUTION DES SÉPULTURES MÉRIDIONALES}

\section{LE POIDS DES TRADITIONS DU NÉOLITHIQUE FINAL}

Le poids des traditions funéraires du Néolithique final est incontestable. En premier lieu, moins de $10 \%$ des sépultures livrant du mobilier campaniforme relève de manifestations individuelles ou apparentées, et ce faible taux ne peut être imputé à un unique biais de la recherche.

Pour la zone méditerranéenne et bas-rhodanienne, la persistance des coffres lithiques constitue également un trait archaïsant, ce qui est aussi le cas des nombreuses associations «plurielles", parfois liées à différentes manipulations évoquant la gestion des dépôts collectifs.

Les différents éléments du mobilier archéologique déposé dans les tombes ne dénotent pas un changement radical dans les pratiques funéraires. Des récipients, des armes et outils ou des parures sont des éléments communs dans les sépultures de la fin du Néolithique.

\section{LES SPÉCIFICITÉS CAMPANIFORMES}

Ces traits archaïsants, cependant, se mêlent souvent en des associations originales et s'inscrivent dans une évolution constatée par ailleurs à plus grande échelle.

Ainsi, les coffres lithiques du Campaniforme et du Bronze ancien I sont généralement de petite taille; ils sont enterrés et ne possèdent plus d'accès permanent. Leur accès est vertical et parfois même bloqué par un cairn ou un tertre.

Les coffres en matériaux périssables rigides semblent prendre le relais dans les phases plus évoluées de la période, comme aux Juilléras. Ils s'insèrent dans des fosses spécifiquement funéraires, bien que certaines fosses domestiques soient encore épisodiquement détournées à cet usage très répandu sur les sites néolithiques des grandes terrasses alluviales. C'est là, aussi, que s'amorce le développement des manifestations de caractères «nécropolaires » sous la forme de cellules d'inhumations dispersées (comme pour le Bronze ancien auvergnat), dont les relations avec l'habitat ne peuvent être pour l'instant réellement appréhendées. Leurs fondations pourraient être étroitement liées au Campaniforme: en ce sens, cette manifestation et/ou culture participe bien au morcellement (phase précoce), puis à l'éclatement (phase tardive) des ensembles collectifs.

Les choix architecturaux, qui se superposent à certaines pratiques, restent donc relativement inféodés aux contextes culturels antérieurs et aux environnements naturels. On perçoit cependant que la nouvelle conjugaison de ces traits s'inspire de ce courant porté par certaines valeurs véhiculées à grande échelle (dont les significations initiales peuvent néanmoins se révéler plus ou moins abstraites pour les populations locales).

Comme nous l'avons dit, cette transformation s'exprime à travers la tendance à clore, enterrer et individualiser espaces et dotations funéraires, mais aussi à travers des composantes plus spécifiques au courant campaniforme: tumulus ou tertres (10 à 12 occurrences), tombes individuelles isolées mais surtout valorisées au sein des aires d'habitat. On pense naturellement à la sépulture de La Fare et celle de la grotte Murée. Pour une phase encore plus évoluée, on peut aussi proposer les coffres lithiques du site G. Besse II-5, de Gigondas et peut-être le coffre 1 des Juilléras (dans la mesure où il est le seul de ce type pour l'ensemble), ainsi que la sépulture "d'archer " en coffre périssable de La Gravière Peer II, en Auvergne. 
Le déficit de mobilier au cours de cette phase demeure tout de même un obstacle à la définition retenue ici, mais rappelons-nous que des ré-interventions sont parfois attestées.

Si les types de mobilier reconnus dans les sépultures campaniformes ne sont pas novateurs, la spécificité campaniforme pourrait être mise en évidence par la place particulière accordée d'une manière générale à la vaisselle de consommation et au gobelet en particulier. Mais cette observation est surtout validée par l'examen des sépultures collectives d'usage ancien, où le Campaniforme est révélé par la présence de ces céramiques spécifiques alors que les dépôts du Néolithique final concernaient plutôt des outils, des armes et des parures.

\section{GÉOGRAPHIE ET DOMAINES D'INFLUENCE}

À l'échelle du grand sud de la France pris en compte dans cette étude, le très faible nombre de sépultures individuelles et plurielles connues permet cependant d'observer quatre groupes distincts : un ensemble atlan- tique, entre Loire et Gironde, qui comprend huit tombes; un ensemble Massif central comprenant quatre tombes; un ensemble Val de Saône avec trois sépultures et un dernier ensemble méditerranéen et bas-rhodanien - le plus important - avec trente structures funéraires. Les zones vides demeurent très étendues, surtout dans le sud-ouest de la France.

Sur le plan des architectures ou des pratiques, la question des territoires et des influences reste peu discernable. La présence de tertre pourrait à la fois évoquer certains tumulus nord-orientaux, mais aussi des sépultures fini-néolithiques de la façade atlantique et des domaines pyrénéens, ce fait traduisant davantage une ambiance que des courants individualisés.

Pour toutes les phases chronologiques, le mobilier funéraire correspond naturellement à la fois aux traditions régionales et à des échanges ou de simples contacts avec d'autres régions. Le faible nombre de tombes ne permet pas d'observer des spécificités géographiques dans les assemblages ou de mettre en relation certaines sépultures avec d'autres traditions particulières.
Au terme de ce bilan ${ }^{29}$, nous constatons que les sépultures individuelles sont à la fois très rares et très diverses pendant tout le cycle campaniforme et au début de l'âge du Bronze dans cette grande moitié sud de la France.

En dépit de leur augmentation régulière, on ne peut observer actuellement un réel développement de ces structures, car si les sépultures du Campaniforme ancien demeurent peu nombreuses, la phase récente ne se distingue pas par leur accroissement significatif, ce qui est aussi le cas de la phase tardive essentiellement représentée par une seule cellule d'inhumation. Finalement, au Bronze ancien I, le nombre de tombes ne semble pas progresser non plus de façon spectaculaire.

C'est bien encore la tradition très forte de la sépulture collective, issue des périodes antérieures, qui marque les pratiques funéraires de cette période, en l'état actuel des connaissances. Mais si le fait d'être

29. Nous tenons à remercier tout particulièrement F. Ducreux, J.-L. Espérou, V. Georges, R. Labeaune et C. Vermeulen, pour les données et la documentation parfois inédite qu'ils nous ont aimablement communiquées. inhumé dans le caveau de la collectivité est si important pour les sociétés de la fin du Néolithique au début de l'âge du Bronze, pourquoi existe-t-il quelques sépultures individuelles? Correspondent-elles à une pratique émergeante? Ce que ne confirment pas les observations actuelles sur l'évolution du nombre de tombes. Cette pratique serait-elle liée à des statuts particuliers? Nous ne pouvons affirmer aujourd'hui qu'il y a des discriminations par le sexe ou par l'âge et il demeure difficile de reconnaître parmi ce corpus très diversifié le statut ou l'origine des individus inhumés : «chefs », « riches » ou " étrangers ».

Une réflexion sur la recherche elle-même pourrait cependant amener à infléchir ces idées. Il semble en effet que si les sépultures collectives en cavité ou monument sont connues et fouillées depuis déjà longtemps, les sépultures individuelles plates ou dont le tertre a été arasé, pouvant être isolées ou situées en marge de l'habitat, relèvent de découvertes fortuites et surtout de fouilles pratiquées lors des décapages extensifs liés à l'archéologie préventive. Une quinzaine de tombes seulement sur les quarante-cinq recensées ici ont été découvertes ou fouillées antérieurement aux années 1990, et plusieurs d'entre elles dans des contextes pré- 
ventifs. Le corpus a ainsi été multiplié par trois en moins de vingt ans. Il est donc très probable que les découvertes s'intensifient dans les années à venir, comme cela se pourrait également pour les tombes se rattachant aux cultures antérieures de la fin du Néolithique, nous amenant ainsi à rééquilibrer nos connaissances et à reconsidérer peut-être l'évolution de ces pratiques funéraires. 